\title{
Transoral Laser Microsurgery for Early Glottic Carcinoma
}

\section{June RJ Lin* and Eitan Prisman}

Division of Otolaryngology, Head and Neck Surgery, Vancouver General Hospital, University of British Columbia, Vancouver, BC, Canada

*Corresponding author: June RJ Lin, Resident Physician, University of British Columbia, Otolaryngology - Head and Neck Surgery, Gordon and Leslie Diamond Health Care Centre, 4th. Floor Rm. 4299B 2775 Laurel St Vancouver, BC V6B6J7, Canada, Tel: 16045619854, E-mail: jrlin13@gmail.com

Rec date: Apr 18, 2014; Acc date: Jul 30, 2014; Pub date: Aug 01, 2014

Copyright: (C) 2014 Lin JRJ, et al. This is an open-access article distributed under the terms of the Creative Commons Attribution License, which permits unrestricted use, distribution, and reproduction in any medium, provided the original author and source are credited.

\begin{abstract}
The application of laser technology to otolaryngology - head and neck surgery has accelerated the development of transoral laser microsurgery, which has a wide range of application in this surgical specialty today. The aim of this paper is to provide a general overview of the surgical techniques of transoral laser microsurgery, particularly in the treatment of early glottic carcinoma.
\end{abstract}

Keywords: Transoral laser microsurgery; Early glottic carcinoma; laser

\section{Early Glottic Carcinoma}

Early glottic carcinoma emcompasses a wide spectrum of disease from moderate dysplasia to carcinoma in situ as well as T1 or T2 tumors without nodal or distant metastases (Figure 1). The ideal management of this disease is one that a) provides a definitive pathologic diagnosis, b) is most likely to result in a cure and c) maximizing vocal function without impairing deglutition or breathing. Treatment options include external beam radiotherapy, open partial laryngectomy or transoral laser excision, all of which have comparable oncologic outcomes (Table 1). A unique advantage of transoral laser microsurgery (TLM) is that it offers a definitive pathological diagnosis using a precise and hemostatic instrument under microscopic visualization combined with the opportunity to preserves vocal function. Furthermore, in patients who develop a local recurrence or a second primary in the head and neck region the array of treatment options remain viable.

Radiotherapy is an alternative option to TLM. For early stage disease, radiation is usually indicated for patients with difficult endoscopic exposure, those that are not suitable for surgery or those with multiple recurrences following endoscopic excision. Surgical salvage following radiotherapy in the form of transoral laser procedures or partial laryngeal surgery are challenging and are associated with a greater risk of wound complications including chondronecrosis and pharyngocutaneous fistula and are generally not recommended. Therefore total laryngectomy is most often the only surgical salvage, with recent evidence promoting the use of vascularized free tissue transfer to buttress the pharyngeal reconstruction to augment the increased risk pharyngocutaneous fistula in previously radiated tissue [1].

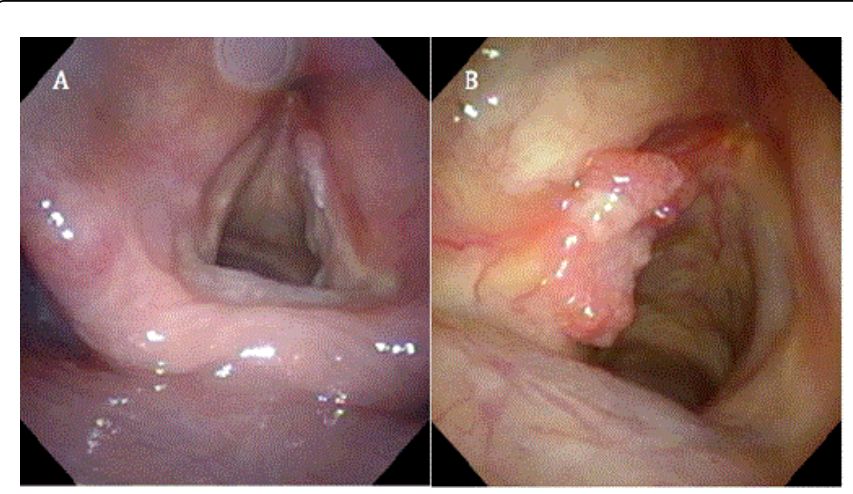

Figure 1: Malignant laryngeal diseases: (A) Right T1 glottic carcinoma; (B) Left T2 glottic carcinoma

\begin{tabular}{|l|l|l|l|}
\hline & TLM & Radiotherapy & Open partial laryngectomy \\
\hline 5-year Local control rate & $77-92 \%[3]$ & $\begin{array}{l}\text { T1-81-90\% [4-6] } \\
\text { T2-64-87\% [4,7] }\end{array}$ & $90-98 \%[8,9]$ \\
\hline 5-year laryngeal preservation rate & $90-99 \%[3]$ & $\begin{array}{l}\text { T1-90-98\% [4-6] } \\
\text { T2 - 75-87\% [4,7] }\end{array}$ & $93-98 \%[8,9]$ \\
\hline
\end{tabular}

Table 1: Oncologic outcomes of TLM, radiotherapy and open partial laryngectomy

Open partial laryngectomy, including vertical hemilaryngectomy, allows for the treatment of larger glottic tumors that may not be amenable to TLM, while preserving critical components of native laryngeal structures. However, in light of the emergence of TLM which has readily addressed early stage larynx cancer, they are not commonly employed [1]. Furthermore, partial laryngeal surgery often requires at least a temporary tracheotomy and may be associated with persistent 
aspiration, failure to decannulate and suboptimal voice outcome [2] (Figure 1 and Table 1).

\section{Pretreatment planning}

Patients presenting with a glottic lesion require a complete workup and when a laryngeal carcinoma is diagnosed this must be appropriately staged. This includes a thorough history and physical examination including neck palpation to rule out metastatic adenopathy and a dedicated flexible fiberoptic laryngoscopy. The latter allows direct visualization of the glottis to examine the extent of the tumor and cord mobility. Combined with laryngeal stroboscopy, this provides unprecedented information regarding impaired mucosal wave which suggests the lesion has infiltrated deeper into the vocal fold structures and would therefore necessitate a deeper excision. Cord fixation upstages the primary tumor to T3, which significantly complicates an oncologically sound resection using transoral laser microsurgery.

Biopsy of the suspected tumor may be performed in the office via a channeled fiberoptic laryngoscope or in the operating room via a suspension laryngoscopy. Fine needle aspiration biopsy of any palpable neck nodes must be performed to confirm neck metastases. Imaging has a limited role in the pretreatment planning of early glottic carcinomas and is controversial except in bulky T2 lesions [10]. However at our institution, any patient with T1 or T2 glottic carcinoma will undergo computed tomography (CT) to further assess the extent of disease that may not be clinically evident. In addition, positron emission tomography (PET-CT) may be an alternative to traditional panendoscopy to evaluate for second primaries or distant metastasis [11].

\section{Cordectomy classification for early glottic cancer}

The European Laryngological Society (ELS) proposed a classification system in 1999 of endoscopic laryngeal cordectomies [12]. A thorough understanding of the five histologic layers of the vocal fold is paramount in order to comprehend the classification system. From superficial to deep, these layers are the stratified squamous epithelium; the superficial lamina propria, also known as Reinke's space; the intermediate lamina propria; the deep lamina propria; and the vocalis muscle (Figure 2) [13]. The combination of intermediate and deep lamina propria is referred to as the vocal ligament. The aforementioned ELS classification system is primarily based on this layered structure of the vocal folds. It includes (1) Type I, or subepithelial cordectomy, (2) Type II, or subligamentous cordectomy, (3) Type III, or transmuscular cordectomy, (4) Type IV, or total cordectomy, (5) Type V, or extended cordectomy, and (6) Type VI, or anterior commissurectomy with bilateral anterior cordectomy.

Type I cordectomy involves the removal of the stratified squamous epithelium of the vocal fold with or without the superficial lamina propria. This allows full thickness examination of the vocal fold epithelium, which is useful in the diagnosis of dysplasia and carcinoma in situ. If the cancer has infiltrated the deeper layers of the vocal fold, which can be predicted based on stroboscopic examination of the vocal folds, then a type II or type III cordectomy should be performed. A type II cordectomy removes the vocal ligament in addition to the epithelium and superficial lamina propria. A type III cordectomy extends the resection a plane deeper to include removing part of the vocalis muscle. If the tumor involves the entire vocal fold, then a type
IV cordectomy is performed, which includes and excision of the vocal fold from the vocal process posteriorly to the anterior commissure. Extended cordectomies are variations tailored towards tumors that have extended into the contralateral vocal fold, ipsilateral arytenoid cartilage, subglottis or laryngeal ventricle. The purpose of this classification is to compare post-operative results following different types of cordectomies that are based on the extent of the patient's glottic carcinoma. It also serves to define and standardize the terminology of cordectomies to facilitate teaching and training surgeons not yet familiar with this type of operation.

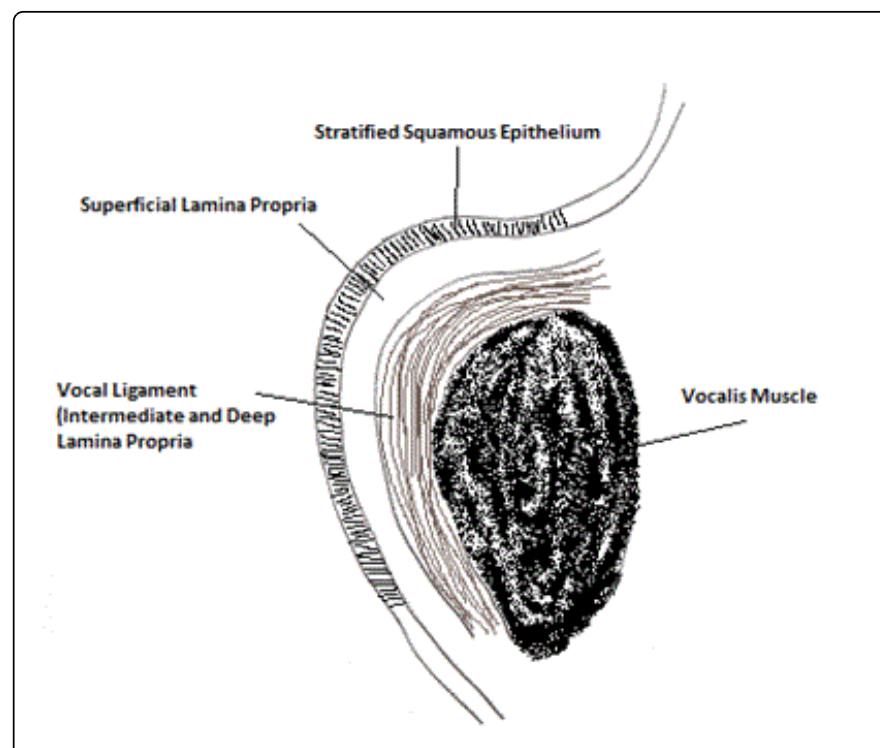

Figure 2: Five histologic layers of the vocal fold

\section{Transoral Laser Microsurgery}

The utility of transoral laser microsurgery in the management of carcinomas in the upper aerodigestive tract was largely popularized by Wolfgang Steiner in the 1980s [14-16]. Today the range of indications has significantly expanded in the realm of head and neck oncology, encompassing the treatment of early and some advanced oropharyngeal, supraglottic, glottic, hypopharyngeal and tracheal tumors. Laser technology has been a key element in the development of endolaryngeal surgery over the past 30 years particularly as an application to treat malignant laryngeal diseases. The history of laser technology in the medical field began with the development of a prototype ruby laser, used to treat a retinal tumor in 1961 [17]. Innovations such as the surgical microscope and general endotracheal anesthesia led to the introduction of the carbon dioxide $\left(\mathrm{CO}_{2}\right)$ laser to the surgical field. In 1967, Thomas Polanyi successfully tested the $\mathrm{CO}_{2}$ laser in human cadaver larynxes [18]. New and innovative uses of the technology continued to evolve, a major advancement being the coupling of the binocular operating microscope with a laser delivery system. Geza Jako reported the first successful use of this equipment in a microlaryngoscopy in 1972 and found that the destruction of specific areas of the vocal cord was feasible and safe in his canine model [19]. Other laser applications in Otolaryngology have been described in detail in other review articles [20,21]. 


\section{Use of $\mathrm{CO}_{2}$ laser in transoral laser microsurgery}

The $\mathrm{CO}_{2}$ laser is the workhorse of laryngologic surgeries in most centers. It has a wavelength of $10,600 \mathrm{~nm}$ on the infrared spectrum, which is preferentially absorbed by water. As a result, the $\mathrm{CO}_{2}$ laser has the advantage of being absorbed by all biologic tissues and is independent of tissue color. The beam itself is invisible, requiring a separate aiming beam for accuracy. Resections are extremely precise due to the aiming beam's spot size of millimeters in diameter (Figure 3 ). Because the target area is so small, the progress of tissue destruction can be carefully monitored. Furthermore, the hemostatic effect of laser energy during tissue destruction maximizes visibility.

Laser power density is a critical parameter in TLM and is a function of the distance of the laser from the tissue, the laser spot size and the power of the laser. Power, energy setting, spot size and duration of the laser procedure have a major role in the thermal effect on the tissue. There are two main modes of delivery of a $\mathrm{CO}_{2}$ laser: continuous wave and pulsed wave. Continuous wave constantly transmits energy to the field. The laser is on as long as the foot pedal is pressed. In the pulsed mode, there is an intermittent application of energy to the laser medium, causing pulses of laser emission. This allows for tissue cooling in between pulses, reducing thermal diffusion. Continuous wave mode is better for coagulation whereas pulsed mode is better for cutting. When working in the larynx, the power settings should be kept low to allow the surgeon to use a smaller spot size, without delivering too large an energy level per unit area. At our institution, initial settings on the $\mathrm{CO}_{2}$ laser is usually started at a power of up to 10 watts, and a spot size of $0.2-0.7 \mathrm{~mm}$. This can be adjusted accordingly during the procedure. Increasing watt energy and defocusing spot size (i.e. making it bigger) simultaneously can maintain the same power density. This helps to spread the energy over a larger surface, which is useful in coagulation or vaporization rather than cutting. If the watt energy is increased without defocusing the spot size, the laser beam becomes more concentrated and will thus penetrate into deeper tissue.

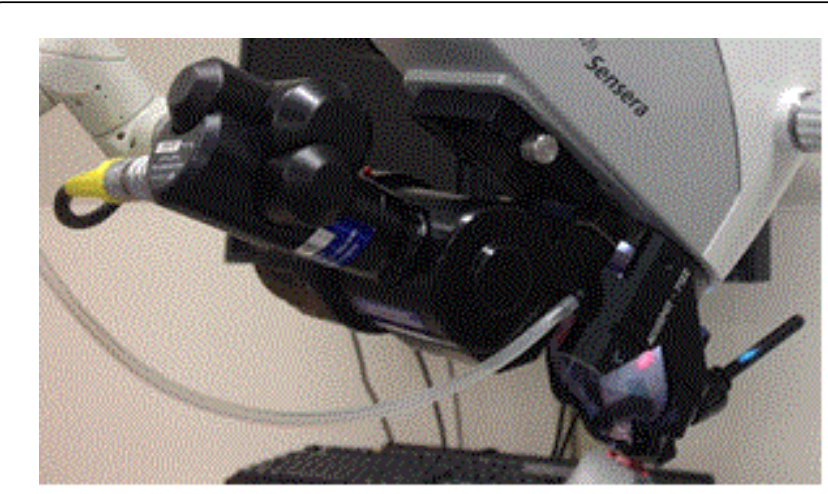

Figure 3: A micromanipulator which couples the $\mathrm{CO}_{2}$ laser and the operating microscope. Note the joystick on the right-hand side, which controls the aiming beam of the laser

\section{Anesthesia}

TLM should be performed under general anesthesia with complete muscle relaxation. An alternative is to use total intravenous anesthesia with the patient spontaneously breathing and is less commonly applied. Controlled intubation and extubation is essential to the procedure and the surgeon should be present during anesthesia induction and exit to monitor the situation and be available to assist the anesthetist should the situation arise. Preoperative discussion with the anesthetist regarding the location and nature of the glottic lesion may also reduce the likelihood of vocal fold injury. Topical administration of lidocaine is essential to decrease the risk of both intraoperative and postoperative laryngospasm and unnecessary hemodynamic instability. Ventilation options include endotracheal intubation as well as jet ventilation via the rigid laryngoscope or a jet ventilation needle.

The choice of ventilation modality will depend on the position of the lesion, the expected length of the procedure, and comfort of the surgeon and anesthesiologist. From the author's experience, the best ventilation option is with a small endotracheal tube, size 5.0 or 5.5, with laser shield where required. This provides ventilation while securing the airway and protects against aspiration. In cases where the endotracheal tube may obstruct the field of view (e.g. lesions at the free edge of the true vocal folds, or subglottic stenosis), or procedures expected to be relatively short, a jet ventilation method is preferred.

\section{Suspension laryngoscopy}

Patients should be positioned in the Boyce-Jackson position, also known as the "sniffing" position for TLM. This is accomplished by having the neck flexed relative to the chest and the head extended relative to the neck (Figure 4). This maneuver should place the larynx along a longitudinal vector with the oropharynx, aiding its visualization. Additional methods of optimizing glottic visualization include adding more pillows or padding behind the patient's head or flexing the neck further onto the chest to lift the head off the operating table. A shoulder roll may help improve visualization however it is not always necessary. Once the patient has been optimally positioned, a laryngoscope can be inserted and suspended. Adequate position is crucial to successful visualization and an oncologically complete and a traumatic surgery. A sufficient amount of time should be dedicated to this step and therefore in situations where this may be challenging, intubating the patient can facilitate a secure airway and provide ample time to optimize laryngeal visualization. There are few contraindications to TLM, including cervical spine disease or an unfavorable anatomy that preclude the patient from having a suspension laryngoscopy.

There is a varied choice of laryngoscopes available for TLM. The optimal choice will be a function of the patient's anatomy, the position of the lesion and the surgeon's preference. The operating laryngoscope, Dedo, is commonly used in many procedures. The Lindholm scope, which is designed as a supraglottoscope, has a larger diameter, providing a much wider exposure and thus more room for surgical instruments. It also has a longer superior lip that helps lift the epiglottis out of the way during insertion. The downside of using the Lindholm scope is that it can be difficult to insert and may cause more trauma, given its size. The anterior commissure scopes are great for patients with difficult airways however they do not provide binocular vision under the microscope and offer little room for operating instruments due to their small caliber. During insertion of the laryngoscope, it is important to unfurl the epiglottis and have it lie flat on top of the superior surface of the laryngoscope. This serves two purposes. The first is to be less traumatic on the epiglottis. The second is to allow more room for the laryngoscope to be advanced into the endolaryx to maximize exposure. After the laryngoscope is suspended, the exposure of the glottis, especially the anterior glottis, can be further optimized by applying counter external pressure using a piece of tape 
across the anterior neck onto both edges of the operating table. Telescopic examination of the glottis and subglottis should be performed using 0 -degree or angled telescopes for photodocumentation and surgical planning.

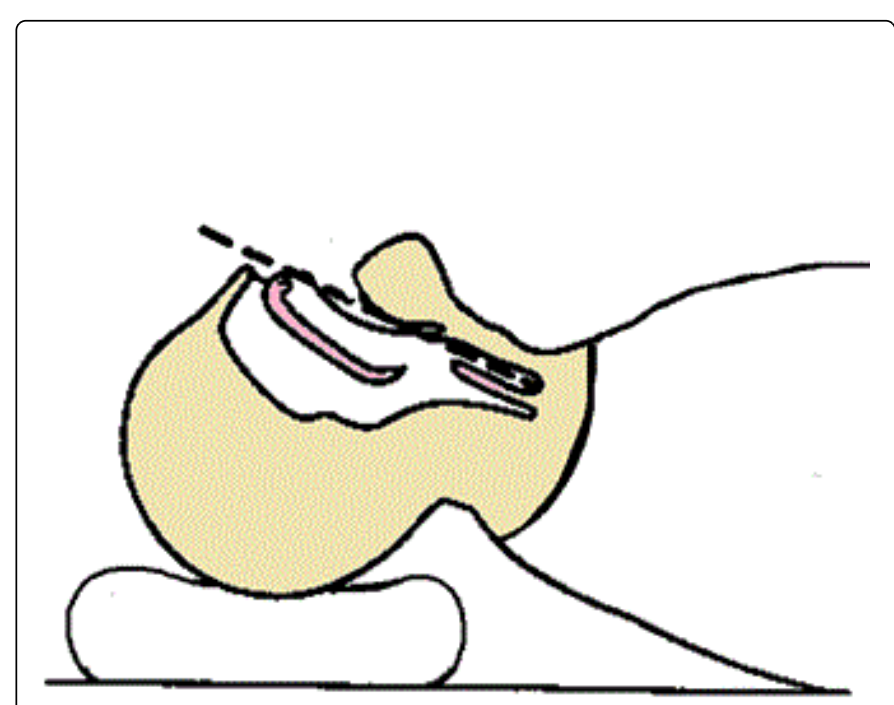

Figure 4: The "sniffing" position

\section{Surgical technique}

Once the preceding steps are done, the laser-coupled microscope can be brought into the surgical field. The laser should be kept at low power settings ranging from 1 to $3 \mathrm{~W}$ at pulsed wave mode. A smoke evacuator can be connected to the rigid laryngoscope via suction tubing to minimize laser plume. Saline is injected into the superficial lamina propria for hydrodissection and to assess the depth of the tumor. If the tumor does not elevate with saline injection, this is an indication that it has infiltrated into deeper layers of the vocal fold. Superficial lesions can be excised en bloc. Resection of larger tumors can be performed via the Steiner technique, which entails making the first incision vertically through the middle of the tumor and extend it down to normal tissue to determine tumor depth. Part of the false vocal cord can be resected in order to improve exposure or be retracted out of the way using a cord spreader. Medial traction is provided by various microlaryngologic instruments to allow lateral dissection. A second incision is then made laterally and posteriorly to remove the posterior half of the tumor. Anterior resection is completed by carrying the dissection laterally and anteriorly to remove the remaining half of the tumor. Orientation of the tumor may be marked by a stitch or positioned and secured on a cork board. A margin of 1 to $2 \mathrm{~mm}$ is usually taken and may be sent for frozen section or permanent section. If the frozen sections are positive, repeat resections are required until subsequent frozen section specimens are negative. If permanent resection margins are positive, a second look procedure can be performed in 6 to 8 weeks and a re-resection performed. Radiation therapy can be used as an alternative.

\section{Complications}

Complications of TLM include tongue edema or numbness, and tooth injury secondary to suspension microlaryngoscopy. Intraoperative hemorrhage can be safely managed using a combination of epinephrine soaked plegdet, defocusing the $\mathrm{CO}_{2}$ laser beam or by using a suction electrocautery. Careful attention to intraoperative hemostasis is critical to prevent the unlikely but dreaded postoperative hemorrhage. Postoperative laryngeal edema is rare, and is often managed conservatively with the administration of perioperative intravenous corticosteroids.

The most dreaded complication of TLM is airway fire. It is important to exercise laser safety precautions in the operating room during these cases. Wet eye pads and towels are used to cover the patient's face to prevent accidental injury to the eyes and skin. Sterile saline or water is made available at the operating table. If jet ventilation is used, careful coordination between the anesthesiologist and surgeon is critical to ensure the patient is not jetted while the laser is on and to minimize the oxygen concentration. In the event of an airway fire, water needs to be poured into the airway immediately and the anesthetist is immediately notified to turn of the oxygen and anethethic gases. The endotracheal tube is then withdrawn and the patient is bag masked. Once the patient's oxygen saturation is stable, a bronchoscopy is performed to assess the extent of mucosal damage and airway can be reestablished by endotracheal intubation. The patient should remain intubated for at least 48 hours while being treated with intravenous antibiotics and corticosteroids. Trial extubation can be attempted 48 hours later followed by a repeat bronchoscopy.

\section{Voice outcome}

Voice quality following TLM for early glottic carcinoma depends on the extent of resection that has been performed. It is typically the case that the more extensive the resection, the worse the voice quality. When comparing vocal function following laser microsurgery and radiotherapy, a meta-analysis identified nine retrospective studies that compared objective voice outcomes in patients undergoing radiotherapy versus laser microsurgery in head-to-head fashion [22]. Although the meta-analysis did trend towards favoring radiotherapy, the results showed no significant difference between the treatment groups. In the author's experience, TLM is associated with minimal functional impairment. Patients are usually discharged from the hospital on the same day tolerating a normal diet. In addition, avoidance of radiotherapy not only preserves salivation and pharyngeal lubrication that assists in speech, but also leaves open this treatment option for possible future recurrences or secondary primary tumors in the head and neck region.

\section{Cost analysis}

Many studies have shown that TLM is a more cost-effective treatment for early glottic carcinoma [23-26]. A study published in 2000 retrospectively collected information on all patients treated for T1N0 glottic carcinoma from 1989 and 1999 [23]. The investigator found that surgery cost approximately $\$ 1900$ per patient whereas radiotherapy cost approximately $\$ 29,000$ per patient, a more than 10 fold difference. The cost benefit analysis does not usually take into account the indirect costs associated with treatment, for example, travel costs and loss of wages from time off work, revealing even stronger economic merit towards TLM as a treatment option. TLM tends to be a day care procedure after which patients can return to work as usual within a few days. This is in contrast to radiotherapy, for which patients will have to take several weeks off from work, which implies a bigger financial loss for the patient. 


\section{Conclusion}

Transoral laser microsurgery has been successfully incorporated as a treatment modality for both benign and malignant laryngeal conditions. It has proven to be a great alternative to partial laryngectomy and radiotherapy, especially for the treatment of early glottic cancer based on oncologic, functional, and even economic considerations. With the ongoing innovations in laser technology and microlaryngoscopic instruments, there is no doubt that laser microsurgery will become more widely adopted over time.

\section{References}

1. Paleri V, Drinnan M, van den Brekel MW, Hinni ML, Bradley PJ, et al. (2014) Vascularized tissue to reduce fistula following salvage total laryngectomy: A systematic review. See comment in PubMed Commons below Laryngoscope 124: 1848-1853.

2. Mandell DL, Woo P, Behin DS, Mojica J, Minasian A, et al. (1999) Videolaryngostroboscopy following vertical partial laryngectomy. See comment in PubMed Commons below Ann Otol Rhinol Laryngol 108: 1061-1067.

3. Mendenhall WM, Werning JW, Hinerman RW, Amdur RJ, Villaret DB (2004) Management of T1-T2 glottic carcinomas. See comment in PubMed Commons below Cancer 100: 1786-1792.

4. Fein DA, Mendenhall WM, Parsons JT, Million RR (1993) T1-T2 squamous cell carcinoma of the glottic larynx treated with radiotherapy: a multivariate analysis of variables potentially influencing local control. See comment in PubMed Commons below Int J Radiat Oncol Biol Phys 25: 605-611.

5. Jørgensen K, Godballe C, Hansen O, Bastholt L (2002) Cancer of the larynx--treatment results after primary radiotherapy with salvage surgery in a series of 1005 patients. See comment in PubMed Commons below Acta Oncol 41: 69-76.

6. Cellai E, Frata P, Magrini SM, Paiar F, Barca R, et al. (2005) Radical radiotherapy for early glottis cancer: results in a series of 1087 patients from two Italian radiation oncology centers I. The case of T1N0 disease. Int J Radiat Oncol Biol Phys 63: 1378-1386.

7. Frata P, Cellai E, Magrini SM, Bonetti B, Vitali E, et al. (2005) Radical radiotherapy for early glottis cancer: results in a series of 1087 patients from two Italian radiation oncology centers II. The case of T2N0 disease. Int J Radiat Oncol Biol Phys 63: 1387-1394.

8. Thomas JV, Olsen KD, Neel HB 3rd, DeSanto LW, Suman VJ (1994) Early glottic carcinoma treated with open laryngeal procedures. See comment in PubMed Commons below Arch Otolaryngol Head Neck Surg 120: 264-268.

9. Giovanni A, Guelfucci B, Gras R, Yu P, Zanaret M (2001) Partial frontolateral laryngectomy with epiglottic reconstruction for management of early-stage glottic carcinoma. See comment in PubMed Commons below Laryngoscope 111: 663-668.

10. Hartl DM, Landry G, Bidault F, Hans S, Julieron M, et al. (2013) CT-scan prediction of thyroid cartilage invasion for early laryngeal squamous cell carcinoma. See comment in PubMed Commons below Eur Arch Otorhinolaryngol 270: 287-291.
11. Haerle SK, Strobel K, Hany TF, Sidler D, Stoeckli SJ (2010) (18)F-FDGPET/CT versus panendoscopy for the detection of synchronous second primary tumors in patients with head and neck squamous cell carcinoma. See comment in PubMed Commons below Head Neck 32: 319-325.

12. Remacle M, Eckel HE, Antonelli A, Brasnu D, Chevalier D, et al. (2000) Endoscopic cordectomy. A proposal for a classification by the Working Committee, European Laryngological Society. See comment in PubMed Commons below Eur Arch Otorhinolaryngol 257: 227-231.

13. Hirano M (1974) Morphological structure of the vocal cord as a vibrator and its variations. See comment in PubMed Commons below Folia Phoniatr (Basel) 26: 89-94.

14. Steiner W (1988) Experience in endoscopic laser surgery of malignant tumours of the upper aero-digestive tract. See comment in PubMed Commons below Adv Otorhinolaryngol 39: 135-144.

15. Steiner W (1993) Results of curative laser microsurgery of laryngeal carcinomas. See comment in PubMed Commons below Am J Otolaryngol 14: 116-121.

16. Ambrosch P (2007) The role of laser microsurgery in the treatment of laryngeal cancer. See comment in PubMed Commons below Curr Opin Otolaryngol Head Neck Surg 15: 82-88.

17. Koester CH, Snitzer E, Campbell CJ, et al. (1962) Experimental laser retina photocoagulation. J Opt Soc Am 52: 607.

18. Polanyi T, Bredermeier HC, Davis TW Jr (1970) A CO2 laser for surgical research. Med Biol Eng Comput 8: 541-548.

19. Jako GJ (1972) Laser surgery of the vocal cords: an experimental study with carbon dioxide laser on dogs. Laryngoscope; 82: 2204-2216.

20. Ossoff RH, Coleman JA, Courey MS, Duncavage JA, Werkhaven JA, et al. (1994) Clinical applications of lasers in otolaryngology--head and neck surgery. See comment in PubMed Commons below Lasers Surg Med 15: 217-248.

21. Reinisch L, Ossoff RH (1996) Laser applications in otolaryngology. Conclusion and future outlook. See comment in PubMed Commons below Otolaryngol Clin North Am 29: 1079-1085.

22. Higgins KM, Shah MD, Ogaick MJ, Enepekides D (2009) Treatment of early-stage glottic cancer: meta-analysis comparison of laser excision versus radiotherapy. See comment in PubMed Commons below J Otolaryngol Head Neck Surg 38: 603-612.

23. Grégoire V, Hamoir M, Rosier JF, Counoy H, Eeckhoudt L, et al. (1999) Cost-minimization analysis of treatment options for T1N0 glottic squamous cell carcinoma: comparison between external radiotherapy, laser microsurgery and partial laryngectomy. Radiother Oncol 53: 1-13.

24. Myers EN, Wagner RL, Johnson JT (1994) Microlaryngoscopic surgery for T1 glottic lesions: a cost-effective option. See comment in PubMed Commons below Ann Otol Rhinol Laryngol 103: 28-30.

25. Foote RL, Buskirk SJ, Grado GL, Bonner JA (1997) Has radiotherapy become too expensive to be considered a treatment option for early glottic cancer? See comment in PubMed Commons below Head Neck 19: 692-700.

26. Brandenburg JH (2001) Laser cordotomy versus radiotherapy: an objective cost analysis. See comment in PubMed Commons below Ann Otol Rhinol Laryngol 110: 312-318. 